\title{
Upper-Extremity Rehabilitation with NAO Robot
}

\author{
Md Assad-Uz-Zaman, Md Rasedul Islam, Mohammed H. Rahman \\ Bio-Robotics Lab, Mechanical Engineering Department, University of Wisconsin-Milwaukee \\ Milwaukee, USA \\ assaduz2@uwm.edu; islam4@uwm.edu; rahmanmh@uwm.edu
}

\begin{abstract}
Proper functioning of human upper limb is substantially important when it comes to activity of daily livings (ADL). Loss of full or partial mobility in upper limb following stroke would make it difficult to perform ADL. Rehabilitating those who lost motor function is one of the way to get their upper limb in use preforming ADL as it was before. We, therefore, have developed an extensive rehabilitation library using NAO robot in which NAO could demonstrate the exercise therapist wants patient to perform. To demonstrate rehabilitation exercises with $\mathrm{NAO}$, a library of recommended rehabilitation exercises involving shoulder (i.e., abduction/adduction, vertical flexion/extension, and internal/external rotation), and elbow (i.e., flexion/extension) joint movements was formed in Choregraphe (graphical programming interface). In experiments, NAO was maneuvered to instruct and demonstrate the exercises from the library. A complex 'touch and play' game was also developed where NAO plays with the subject that represents a multi-joint movement's exercise.
\end{abstract}

Keywords: Rehabilitation, NAO, Upper extremity impairment, Stroke, Choregraphe.

\section{Introduction}

Upper extremity impairment is very common due to geriatric disorders and/or following a stroke or other conditions such as TBI, SCI, sports, falls, and traumatic injuries. According to the World Health Organization (WHO), a stroke is a sudden ischemic or hemorrhagic interruption in the blood flow supplying oxygen and nutrients to the brain tissue. This event results in brain cell death and consequently in partial loss of neurological function [1]. American heart association reports, approximately 785,000 persons experienced a new or recurrent cerebral vascular accident (CVA) or stroke annually in the United States among which number of deaths estimated at 58000 [2]. The consequences of strokes extend further than patient mortality. The majority of stroke survivors live with long-term disabilities, leading to serious social and economic impacts: it is estimated that stroke cost more than 124.5 billion in United States due to stroke [3]. These numbers will continue to rise as the aging population increases.

Several hypotheses exist as to how upper extremity rehabilitation may be improved. Studies reveal that intensive and repetitive therapies significantly improve motor skill [4]. Note that the passive rehabilitation therapy does not contribute in building muscle but does help to prevent contractures, increasing the range of motion and thus maintains and promotes mobility of the patients [5]. Therefore, once resistance to passive arm movements in individuals has diminished it is essential that they practice active movements. For example, the subjects perform any specific task under the guidance of a physiotherapist or a caregiver. To provide such therapy with a robotic rehabilitation protocol, the robotic devices will guide the subject's movement to complete the specified task. Further studies reveal that enhanced motor learning occurs in the 'active rehabilitation therapy' mode, when patients (independently) practice a variety of functional tasks [6] such as grasping and reaching movements and receive feedback (e.g., visual and haptic feedback) intermittently [7-8].

To assist physically disabled individuals with impaired upper limb function, extensive research has been carried out and ongoing in many branches of robotics, particularly on wearable robots e.g., exoskeletons [9-21]. Although much progress has been made, we are still far from the desired achievement, as existing robots have not yet been able to restore body mobility or function.

To instruct and demonstrate exercise to the people with partial loss of upper extremity functionality, we used NAO robot. NAO humanoid robot can interact with people and able to do complex manoeuvring. A set of daily rehabilitation exercise was built in Choregraphe and implemented as a behaviour in NAO robot. In the next section of this paper, a brief overview on the kinematic model of upper extremity is presented. Details about the NAO robot is explained in section 3 . In 
section 4, rehabilitation exercise library building and behaviour implementation using Choregraphe are presented and finally the paper ends with the conclusion and future research works in section 5 .

\section{Kinematic Model of Upper Extremity}

To develop rehabilitation exercise for human upper extremity, kinematics of upper extremity should be analysed. Our study has focused on human right arm's kinematics. Performing ADL requires human upper extremity to be modeled as a serial robotic manipulator with 7 degree of freedom (Shoulder abduction/adduction, vertical flexion/extension, internal/external rotation, Elbow flexion-extension, Forearm pronation-supination, radial/ulnar deviation, and Wrist flexion/extension). Due to the hardware (constraints) limitation of NAO robot, in this research, we have modeled human upper arm as 4 degree of freedom manipulator (Shoulder abduction/adduction, vertical flexion/extension, internal/external rotation, Elbow flexion-extension). Modified Denavit-Hartenberg (DH) conventions were used to develop the kinematic model of human upper limb and coordinate frames were assigned in every joints [22]. As shown in Figure 1, the joint axes of rotation of the human right upper limb are indicated by dark black arrow heads (i.e., $z_{i}$ ). In this model, joints 1 , and 2 together constitute the shoulder joint, where joint 1 corresponds to abduction/adduction joint 2 represents vertical flexion/extension, joint 3 corresponds to internal/external rotation of the shoulder joint and joint 4 represents the flexion/extension of the elbow joint. The elbow joint is located at a distance $d_{\text {upper_arm }}$ and wrist joint is located at a distance $d_{\text {forearm }}$. The modified DH parameters corresponding to the placement of the link frames (in Figure 1) are summarized in Table 1.

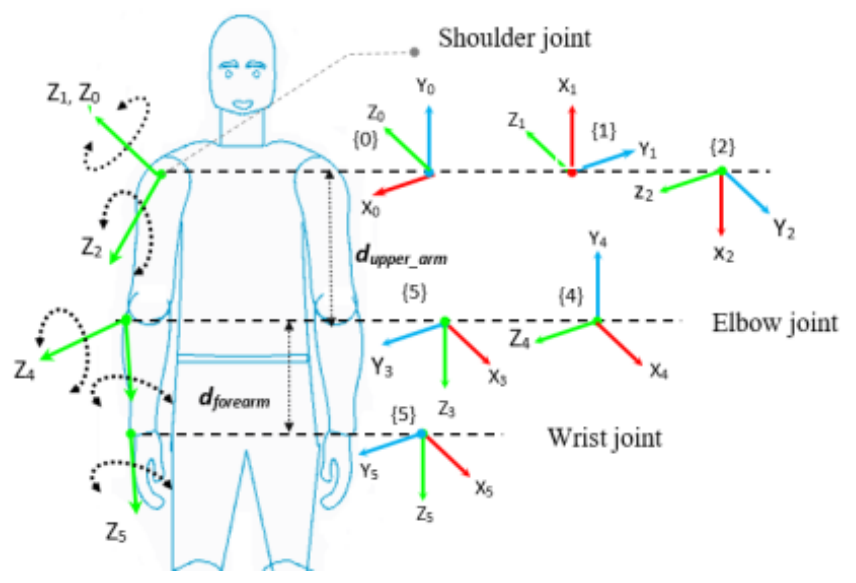

Table 1: Modified DH parameters

\begin{tabular}{|c|l|l|l|l|}
\hline Joint $(\boldsymbol{i})$ & $\boldsymbol{\alpha}_{\boldsymbol{i}-\boldsymbol{1}}$ & $\boldsymbol{d}_{\boldsymbol{i}}$ & $\boldsymbol{a}_{\boldsymbol{i}-\boldsymbol{I}}$ & $\boldsymbol{\theta}_{\boldsymbol{i}}$ \\
\hline 1 & 0 & 0 & 0 & $\theta_{I^{-}}-\pi / 2$ \\
\hline 2 & $\pi / 2$ & 0 & 0 & $\theta_{2}$ \\
\hline 3 & $\pi / 2$ & $d_{\text {upper_arm }}$ & 0 & $\theta_{3}$ \\
\hline 4 & $-\pi / 2$ & 0 & 0 & $\theta_{4}$ \\
\hline 5 & $\pi / 2$ & $d_{\text {forearm }}$ & 0 & 0 \\
\hline
\end{tabular}

Figure 1: Link frame attachments to the human right limb.

We know that the general form of a link transformation that relates frame $\{i\}$ relative to the frame $\{i-1\}$ is:

$$
{ }_{i}^{i-1} T=\left[\begin{array}{cc}
{ }_{i}^{i-1} R^{3 \times 3} & { }_{i}^{i-1} P^{3 \times 1} \\
00^{1 \times 3} & 1
\end{array}\right]
$$

where, ${ }_{i}^{i-1} R$ is the rotation matrix that maps frame $\{i\}$ relative to frame $\{i-1\}$ and can be expressed as:

$$
{ }_{i}^{i-1} R=\left[\begin{array}{ccc}
\cos \theta_{i} & -\sin \theta_{i} & 0 \\
\sin \theta_{i} \cos \alpha_{i-1} & \cos \theta_{i} \cos \alpha_{i-1} & -\sin \alpha_{i-1} \\
\sin \theta_{i} \sin \alpha_{i-1} & \cos \theta_{i} \sin \alpha_{i-1} & \cos \alpha_{i-1}
\end{array}\right]
$$

and, ${ }_{i}^{i-1} P$ is the vector that locates the origin of frame $\{i\}$ relative to frame $\{i-1\}$ and can be expressed as: 


$$
{ }_{i}^{i-1} P=\left[\begin{array}{lll}
a_{i-1} & -s \alpha_{i-1} d_{i} & c \alpha_{i-1} d_{i}
\end{array}\right]^{T}
$$

Using Equations (1), (2) and (3) the individual homogeneous transfer matrix that relates two successive frame (of figure) can be found as:

$$
\begin{aligned}
& { }_{1}^{0} T=\left[\begin{array}{cccc}
\cos \left(\theta_{1}+\frac{\pi}{2}\right) & -\sin \left(\theta_{1}+\frac{\pi}{2}\right) & 0 & 0 \\
\sin \left(\theta_{1}+\frac{\pi}{2}\right)+ & \cos \left(\theta_{1}+\frac{\pi}{2}\right) & 0 & 0 \\
0 & 0 & 1 & 0 \\
0 & 0 & 0 & 1
\end{array}\right] ;{ }_{2}^{1} T=\left[\begin{array}{cccc}
\cos \left(\theta_{2}-\frac{\pi}{2}\right) & -\sin \left(\theta_{2}-\frac{\pi}{2}\right) & 0 & 0 \\
0 & 0 & -1 & 0 \\
\sin \left(\theta_{2}-\frac{\pi}{2}\right) & \cos \left(\theta_{2}-\frac{\pi}{2}\right) & 0 & 0 \\
0 & 0 & 0 & 1
\end{array}\right] \\
& { }_{3}^{2} T=\left[\begin{array}{ccccc}
\cos \theta_{3} & -\sin \theta_{3} & 0 & 0 \\
0 & 0 & -1 & -d_{\text {upper_arm }} \\
\sin \theta_{3} & \cos \theta_{3} & 0 & 0 \\
0 & 0 & 0 & 1
\end{array}\right] ; \quad{ }_{4}^{3} T=\left[\begin{array}{cccc}
\cos \theta_{4} & -\sin \theta_{4} & 0 & 0 \\
0 & 0 & 1 & 0 \\
-\sin \theta_{4} & -\cos \theta_{4} & 0 & 0 \\
0 & 0 & 0 & 1
\end{array}\right] \\
& { }_{5}^{4} T=\left[\begin{array}{cccc}
\cos \theta_{5} & -\sin \theta_{5} & 0 & 0 \\
0 & 0 & -1 & -d_{\text {forearm }} \\
\sin \theta_{5} & \cos \theta_{5} & 0 & 0 \\
0 & 0 & 0 & 1
\end{array}\right]
\end{aligned}
$$

The homogenous transformation matrix that relates frame $\{5\}$ to frame $\{0\}$ can be obtained by multiplying individual transformation matrices.

$$
{ }_{5}^{0} T=\left[{ }_{1}^{0} T \cdot{ }_{2}^{1} T{ }_{3}^{2} T \cdot{ }_{4}^{3} T \cdot{ }_{5}^{4} T\right]
$$

The single transformation matrix thus were found from Equation Error! Reference source not found. represents the positions and orientations of the reference frame attached to the wrist joint (axis 5) with respect to the fixed reference frame $\{0\}$. The equation obtained from this transformation matrix is known as forward kinematics equation. If the joint variable of each joint $\left(\theta_{1}, \theta_{2}, \theta_{3}\right.$, and $\left.\theta_{4}\right)$ is known then wrist joint's position with respect to the base frame $\{0\}$ can be determined using the equation (4).

\section{NAO Robot}

Humanoid robot NAO, developed by Aldebaran Robotics, is one of the most promising autonomous programmable robot. NAO has drawn the attention of researchers and health professionals due to its capability of human like acrobatic movement and communication such as vision, speech, hearing and touch-sensing feature. It has total 25 degree of freedoms (Figure 2) for whole body movement, equipped with two cameras, four microphones, two loud speakers, nine tactile sensors, and eight pressure sensors [23]. In this research, we have used NAO V5. NAO V5 is $574 \mathrm{~mm}$ tall and $275 \mathrm{~mm}$ width with $5.4 \mathrm{~kg}$ body weight. Its upper arm length is $105 \mathrm{~mm}$ and lower arm length is $55.95 \mathrm{~mm}$. Its thigh length 100 $\mathrm{mm}$, tibial length $102.90 \mathrm{~mm}$ and foot height $45.19 \mathrm{~mm}$. In this study, focus remained on upper extremity rehabilitation scheme, in particular, upper arm (right) of the NAO (Figure 3). The range of motion of NAO is slightly different compared to human upper arm range of motion. For instance, its elbow joint range of motion is less compare to the human elbow joint motion. In addition, NAO's shoulder joint roll (abduction/adduction) motion range is smaller compare to that of human shoulder joint motion. On the other hand, its shoulder pitch (vertical flexion/extension) range of motion is larger compare to the human shoulder joint pitch motion. Human upper-extremity joints motions are depicted in Figure 4. 


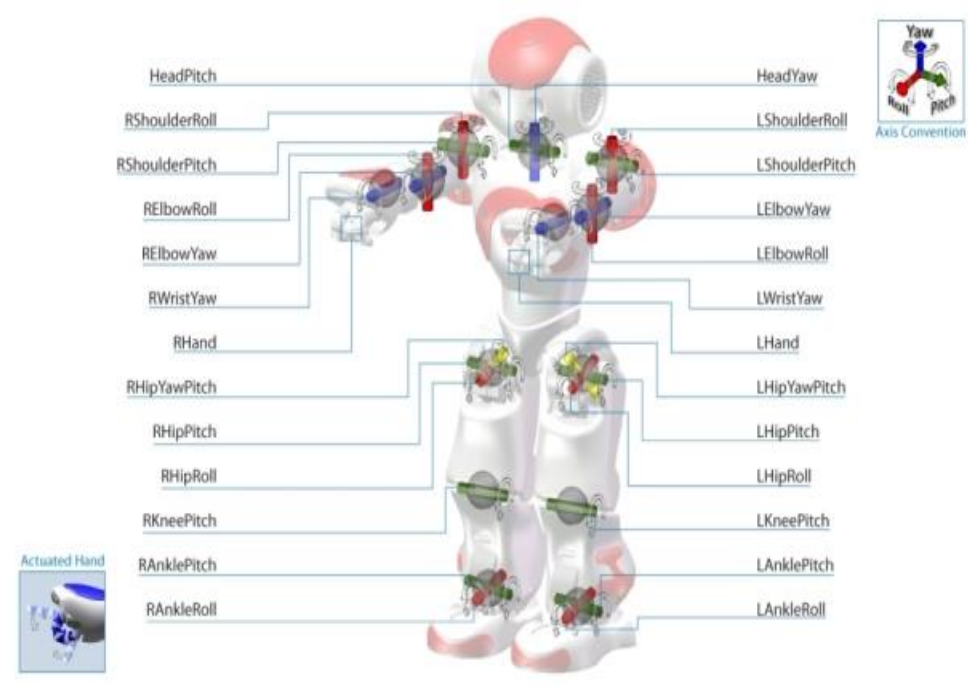

Fig. 2: All joints in NAO robot and initial position [23].
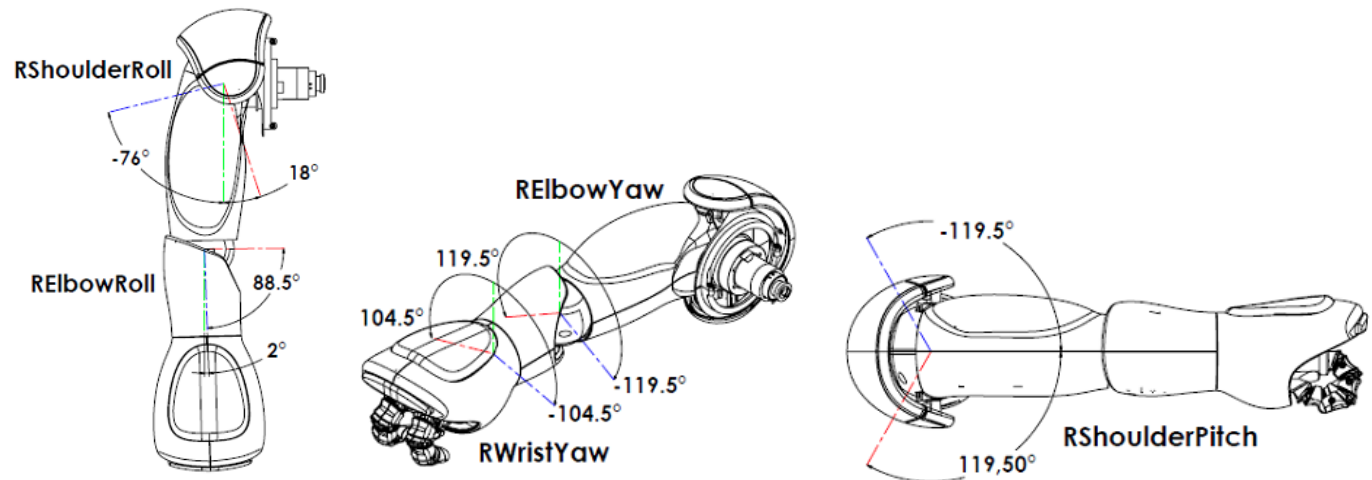

Fig. 3: Right arm joints and range of motions [23].

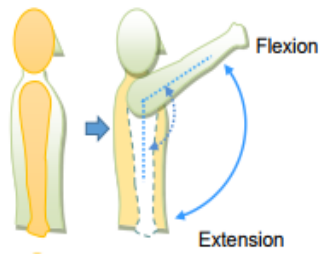

(a)

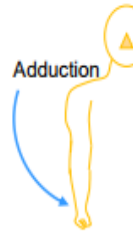

(b)

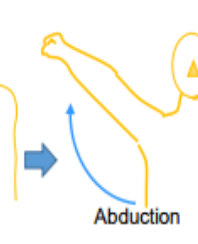

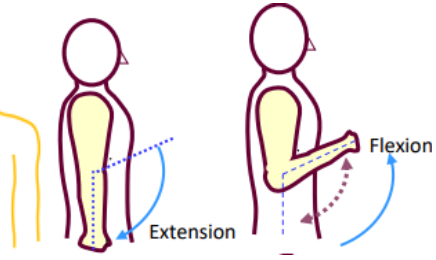

(c)

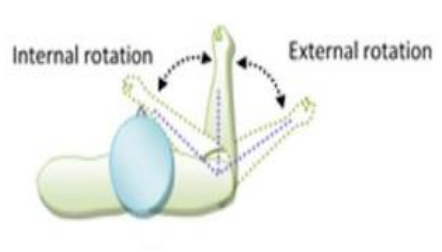

(d)

Fig. 4: General motion. (a) Shoulder Flexion/Extension (shoulder pitch); (b) Shoulder Abduction/Adduction (shoulder roll); (c) Elbow Flexion/Extension (elbow roll) and (d) Shoulder Internal/External rotation (elbow yaw).

Table 2: Upper extremity range of motion of NAO robot and human subject.

\begin{tabular}{|l|l|l|c|}
\hline Joint Name & \multicolumn{1}{|c|}{ Motion } & Range for NAO & Range for human [24] \\
\hline RshoulderPitch & $\begin{array}{l}\text { Right shoulder joint front and back } \\
\text { (Y) }\end{array}$ & -119.5 to 119.5 & -150 to +30 \\
\hline RshoulderRoll & Right shoulder joint right and left (Z) & -76 to 18 & -50 to +180 \\
\hline RElbowRoll & Right elbow joint (Z) & 2 to 88.5 & 0 to +150 \\
\hline RElbowYaw & Right shoulder joint twist (X) & -119.5 to 119.5 & -90 to +15 \\
\hline
\end{tabular}


The behaviour of NAO can be built by using a high level block based programming environment called Choregraphe [25] or by using other programming languages for which appropriate API is available. Choregraphe is a multi-platform desktop application that can create complex behaviours without writing any code. It is actually a graphical programming interface. Different functionalities of NAO are represented here as behaviour blocks. All those blocks provide specific task for NAO. By combining different blocks, one can create new behaviour block. Like LabVIEW and Simulink, Choregraphe use same concept of signal flow and executes the blocks in order they are connected to each other. In addition, Choregraphe provides behaviour control of NAO. One can remove an old behaviour or can add a new behaviour through this. It is very user friendly. Options are available to create new behaviour blocks for advanced functionality (of NAO) in Choregraphe by using programming language python with python API.

\section{Exercise Building and Behaviour Implementation}

To demonstrate rehabilitation exercises with NAO, a library of recommended rehabilitation exercises involving shoulder and elbow joint movements was formed in Choregraphe (graphical programming interface). Choregraphe is high level programming interface for NAO robot. Choregraphe built-in blocks provide access to all sensors and actuators of NAO. In addition, bocks are used to access in NAO's memory. It is also possible to build a new functional block in Choregraphe using programming language Python with the provided SDK. It also offers a virtual NAO in which one can perform simulation of created behaviors. Choregraphe contains different types of blocks. Audio, vision, motion, sensing etc. There has also some advanced built-in blocks in Choregraphe such as speech recognition, face recognition, learning face, detect face etc. Workflow in Choregraphe follows a parent child relation. Each block has input and output ports. They are connected through lines/wires and program flow in sequential order. Another important functional blocks library in Choregraphe is the 'Timeline' block. Using this block, each motor was controlled in a time frame along with executing different other functional blocks. The joint trajectory for different types of motion exercises were generated and set those in a library. In next section, different joint trajectories representing single and multi-joint movement rehabilitation exercises are presented. Choregraphe also provides some flow control blocks such as conditional statement blocks 'if-else', 'for loop', 'switch' etc. These conditional blocks are used to generate a cooperative rehabilitation exercise library.

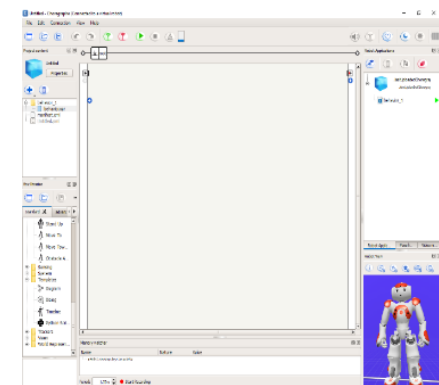

(a)

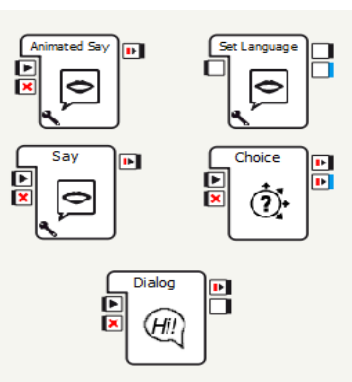

(b)

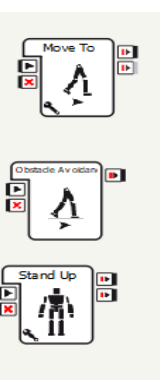

(b)

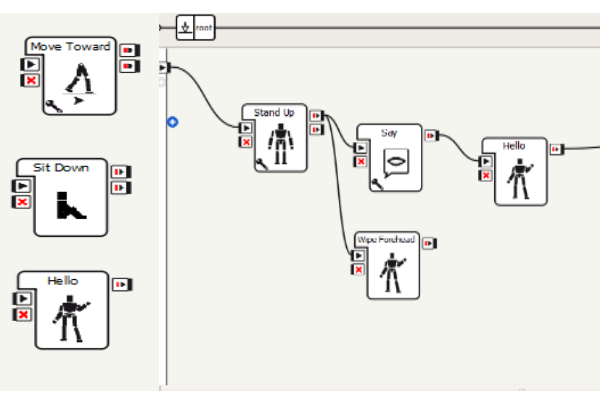

(c)

Fig. 5: (a) Choregraphe programming interface; (b) Functions blocks in Choregraphe; (c) Program flow in Choregraphe.

The exercises formed in library can be grouped under three categories; 'single joint movement', 'multi joint movements', and 'co-operative exercise'. NAO will instruct and demonstrate subjects to perform those exercises. A typical NAO's instruction is given below:

\{

NAO: Hello friend,

NAO: Let's get ready. Stay normal and don't worry. I am here for you mate. Believe me it's going to be fun.

NAO: Anyway, we will do some exercise together

NAO: In this session, I will show you how to do elbow flexion/extension exercise in a minute. After that you will be asked to do perform the exercise...

$\vdots$

\} 
Single joint movement exercises in library include shoulder joint abduction/adduction, shoulder joint vertical flexion/extension, shoulder joint internal/external rotation, and elbow joint flexion/extension motion. Since NAO does not have wrist joint flexion/extension and radial/ulnar deviation, we have excluded wrist joint motions from this study. Figure 7(a) shows the experimental results of shoulder joint abduction/adduction where a coordinated movement of shoulder horizontal and vertical flexion/extension motion were performed. The top plot of Figure 7(a) shows NAO's abduction/adduction angle as a function of time. The bottom plot of Figure 7(a) shows the joint velocity. As shown in Figures 7(a), and (b), the exercise began with NAO's adduction angle $0^{\circ}$ then abduction motion was performed, finally the exercise ends with the NAO's adduction to $0^{\circ}$. Maximum abduction angle observed in this case was $-75^{\circ}$.

Multi joint movement exercises in library include a combination and co-operative movements of shoulder joint (abduction/adduction, vertical flexion/extension, internal/external rotation), and elbow joint (flexion/extension) motion. Reaching movements are widely used and recommended for multi joint movement exercises. A diagonal reaching movement exercise is depicted in Figure 8(a) and (b) respectively. Diagonal reaching movement involves shoulder joint flexion/extension motion, shoulder joint abduction/adduction, and elbow joint flexion/extension motion. Experimental results of diagonal reaching movement showing the elbow and shoulder joint angles are illustrated in Figure 8(b). NAO will instruct the subjects to perform a repetitive motion of this exercise.

Finally, to perform more complex cooperative exercises with NAO, we combined all the functional behaviors (single joint movement exercises, multi join movement exercise, co-operative movement exercise) described earlier. A sample of such cooperative exercise (developed in Choregraphe) is shown in Figure 9.
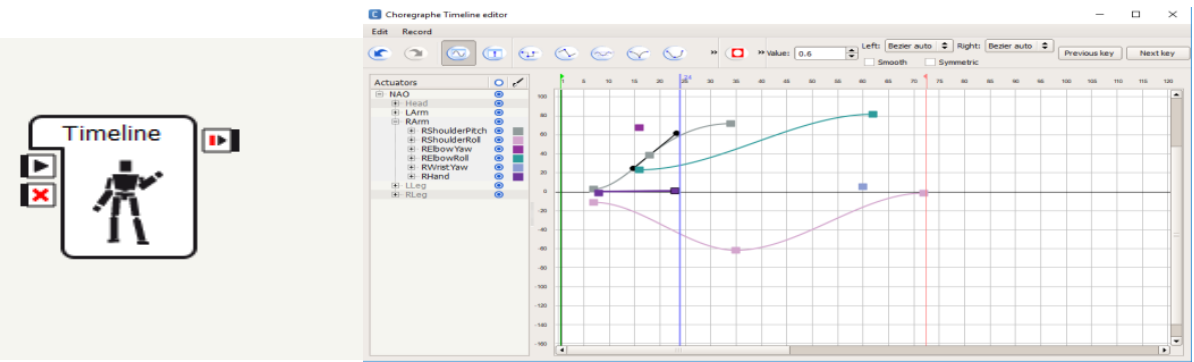

Fig. 6: Timeline block and Motor trajectory control inside the timeline block.
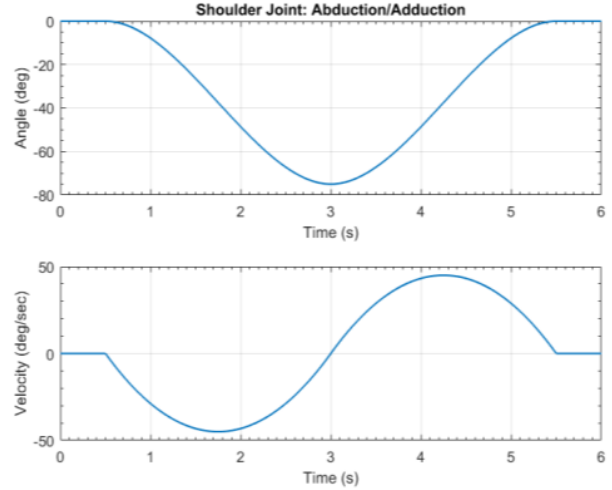

(a)

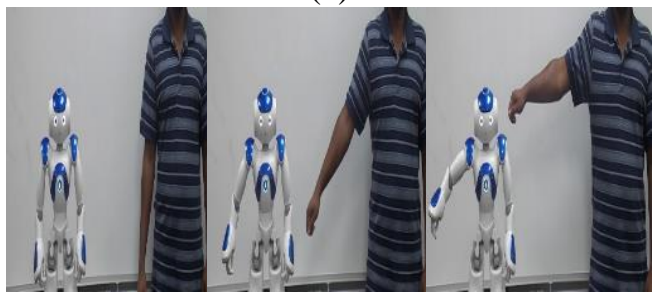

(b)

Fig. 7: (a) Shoulder joint abduction/ adduction motion (joint trajectory and velocity); (b) Abduction/adduction motion of NAO with human. 

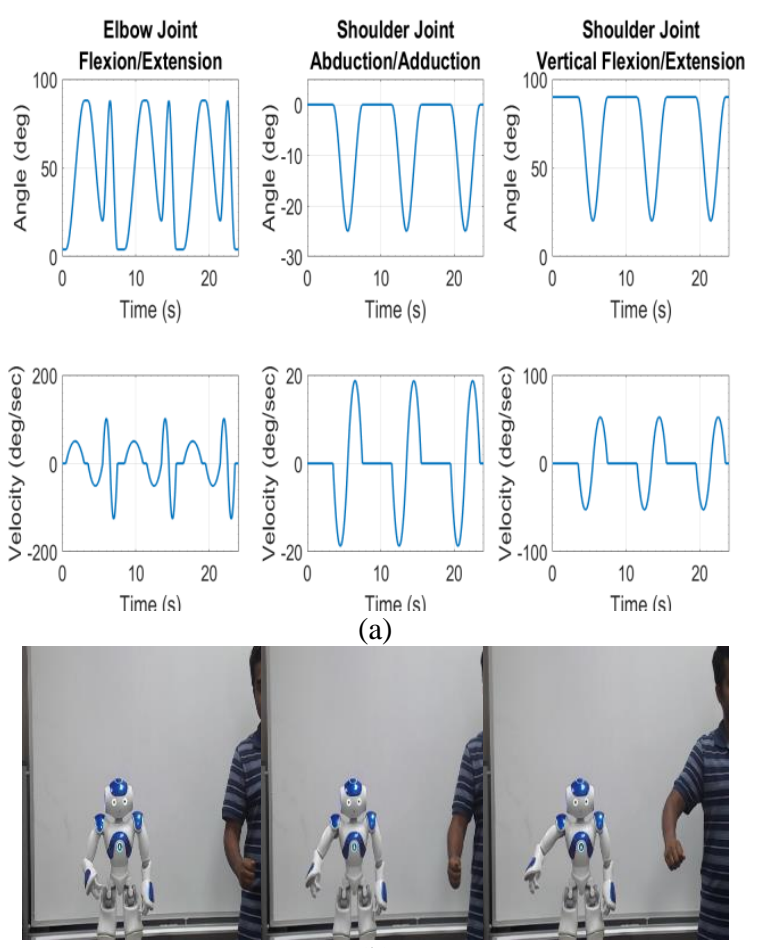

(b)

Fig. 8: (a) Diagonal reaching movements (joint trajectory and velocity); (b) Diagonal reaching movements performed/ demonstrated by NAO.

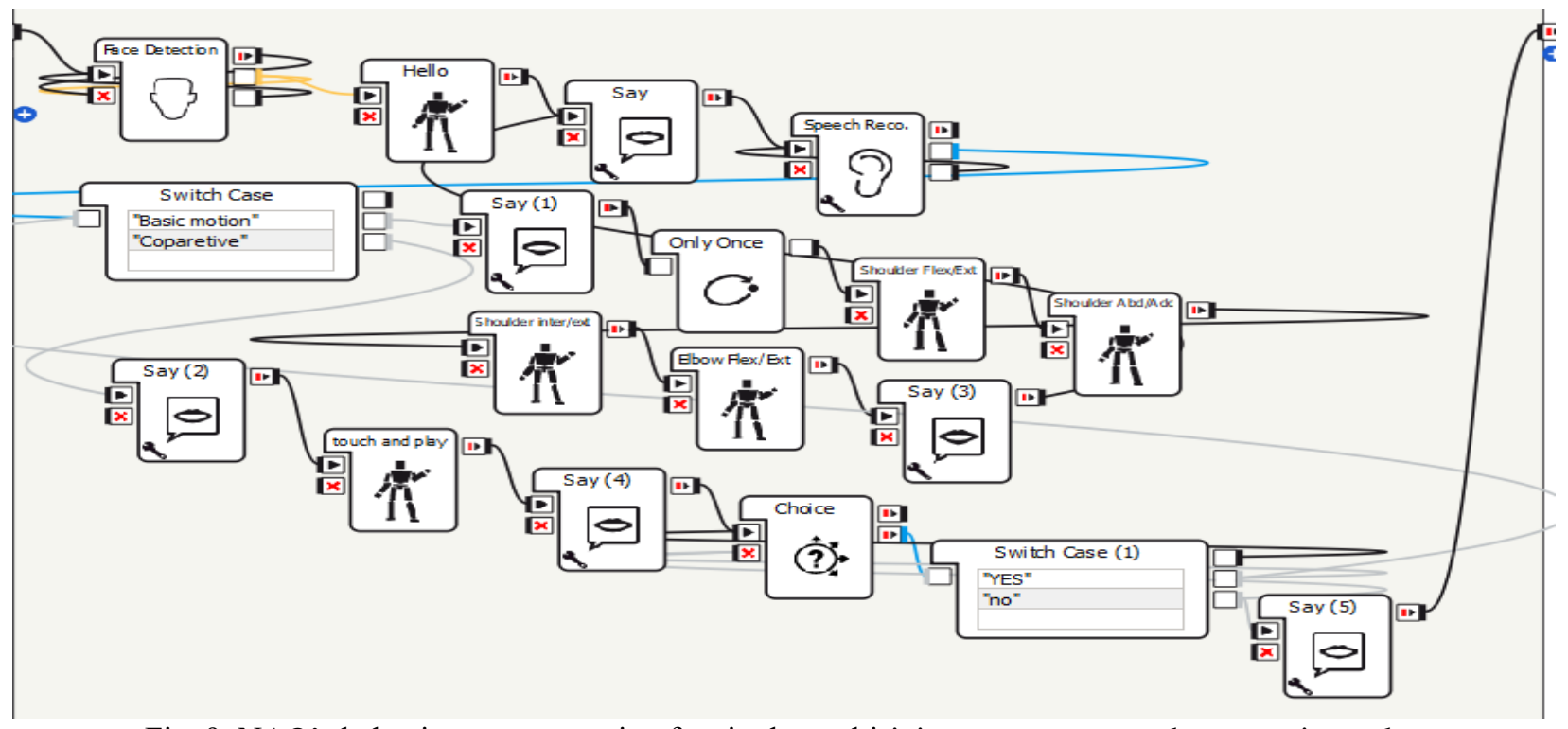

Fig. 9: NAO’s behaviour programming for single, multi-joints movements and cooperative tasks.

\section{Conclusion and Future Work}

Experimental results reveal that NAO can be effectively used to instruct and demonstrate upper-extremity rehabilitation exercises for single and multi-joint movements. Implemented rehabilitation exercise library can be executed anytime using NAO robot. An individual can easily communicate (verbally) with NAO to perform such behavior. Such a robotic system can facilitate a large number of subjects under a single rehabilitation session. Complex cooperative exercise 
can also be developed for NAO robot. Choregraphe provides user friendly environment to introduce new exercises in such library for NAO robot. All this make possible to utilize NAO robot as an effective means for rehabilitation.

To provide robot assisted rehabilitation future projects may include developing an intelligent robot sensor system that can assist therapist to instruct, supervise and demonstrate exercise intelligently. Future studies/works can also be expanded as developing a low cost humanoid robot with more processing power and high durability, NAO has limitations in his processing power and develop a cloud server system of rehabilitation exercises in which individuals can access from anywhere to explore different types of rehabilitation exercises.

\section{References}

[1] World Health Organization. (2017). "Stroke, Cerebrovascular accident." Retrieved November 20, 2017, [Online]. Available: http://www.who.int/topics/cerebrovascular_accident/en/

[2] E. J. Benjamin, M. J. Blaha, S. E. Chiuve, M. Cushman, S. R. Das, R. Deo, et al., Heart Disease and Stroke Statistics - 2017 Update: A Report From the American Heart Association. Circulation, p. 230, 2017.

[3] D. Mozaffarian, E. J. Benjamin, A. S. Go, D. K. Arnett, M. J. Blaha, M. Cushman, S. de Ferranti, J. P. Despres, H. J. Fullerton, V. J. Howard, M. D. Huffman, S. E. Judd, B. M. Kissela, D. T. Lackland, J. H. Lichtman, L. D. Lisabeth, S. Liu, R. H. Mackey, D. B. Matchar, D. K. McGuire, E. R. Mohler, 3rd, C. S. Moy, P. Muntner, M. E. Mussolino, K. Nasir, R. W. Neumar, G. Nichol, L. Palaniappan, D. K. Pandey, M. J. Reeves, C. J. Rodriguez, P. D. Sorlie, J. Stein, A. Towfighi, T. N. Turan, S. S. Virani, J. Z. Willey, D. Woo, R. W. Yeh and M. B. Turner, "Heart disease and stroke statistics - 2015 update: a report from the American Heart Association." Circulation, vol. 131, no. 4, pp. e29$322,2015$.

[4] X. L. Hu, K. Y. Tong, R. Song, X. J. Zheng, K. H. Lui, W. W. F.Leung, S. Ng, S. S. Y. Au-Yeung, "Quantitative evaluation of motor functional recovery process in chronic stroke patients during robot-assisted wrist training," $J$ Electromyogr Kinesiol, vol. 19, no. 4, pp. 639-650, 2009.

[5] D. Wang, (2011), "Physical Therapy Exercises for a Stroke Patient's Arm," (November 15, 2017). [Online]. Available: http://www.livestrong.com/article/312280-physical-therapy-exercises-for-astroke-patients-arm/

[6] C. J. Winstein, A. S. Merians and K. J. Sullivan, "Motor learning after unilateral brain damage," Neuropsychologia, vol. 37, no. 8, pp. 975-987, 1999.

[7] C. J. Winstein, J. P. Miller, S. Blanton, E. Taub, G. Uswatte, D. Morris, D. Nichols and S. Wolf, "Methods for a multisite randomized trial to investigate the effect of constraint-induced movement therapy in improving upper extremity function among adults recovering from a cerebrovascular stroke," Neurorehabil Neural Repair, vol. 17, no. 3, pp. 137-152, 2003.

[8] P. S. Lum, C. G. Burgar and P. C. Shor, "Evidence for improved muscle activation patterns after retraining of reaching movements with the MIME robotic system in subjects with post-stroke hemiparesis," IEEE Transactions on Neural Systems and Rehabilitation Engineering, vol. 12, no. 2, pp. 186-194, 2004.

[9] M. H. Rahman, K. Kiguchi, M. M. Rahman and M. Sasaki, "Robotic exoskeleton for rehabilitation and motion assist," in 1st International Conference on Industrial and Information Systems, ICIIS 2006, pp. 241-246, Peradeniya, Sri lanka, 2006.

[10] P. Garrec, J. P. Friconneau, Y. Measson and Y. Perrot, "ABLE, an innovative transparent exoskeleton for the upperlimb," in 2008 IEEE/RSJ International Conference on Intelligent Robots and Systems, pp. 1483-1488, Piscataway, NJ, USA: IEEE, 2008.

[11] T. Nef, M. Guidali and R. Riener, "ARMin III - arm therapy exoskeleton with an ergonomic shoulder actuation," Applied Bionics and Biomechanics, vol. 6, no. 2, pp. 127-142, 2009.

[12] M. R. Islam, C. Spiewak, M. H. Rahman, R. Fareh, "A Brief Review on Robotic Exoskeletons for Upper Extremity Rehabilitation to Find the Gap between Research Porotype and Commercial Type," Adv Robot Autom, vol. 6, pp. 177, 2017. doi:10.4172/2168-9695.1000177

[13] C.Spiewak, M. R. Islam, M. A.Rahaman, M. H. Rahman, R. Smith, and M. Saad, "Modeling and control of a 4dof robotic assistive device for hand rehabilitation," International Journal of Mechanical, Aerospace, Industrial, Mechatronic and Manufacturing Engineering, vol. 10, no. 8. pp. 1372-1376, 2016. 
[14] B.Brahim, M. H. Rahman, M. Saad, C. Ochoa Luna, and Md. R. Islam, "Sliding Mode Backstepping Control for Upper-Limb Rehabilitation with the ETS-Marse Exoskeleton Robot," RESNA/NCART Conference 2016, Washinton DC, USA, 2016.

[15] Md. R. Islam, Md. Assad-Uz-Zaman, M. H. Rahman, "Motion Control of Robot for passive rehabilitation of human shoulder," UWM's CEAS 2017 poster competition, Milwaukee, WI, USA, 2017.

[16] Md R. Islam, Md A.-Uz-Zaman, C. Spiewak, M. H. Rahman, "Motion Control of a Robotic Device for Passive Rehabilitation of Human Shoulder and Elbow Joint Movement," Great Lakes Biomedical Conference 2017, Milwaukee, WI, USA, 2017.

[17] C. Spiewak, M. R. Islam, M. H. Rahman, R. Smith, M. Saad, "Nonlinear Control of a Robotic Exoskeleton for Hand Rehabilitation," MSOE's IEEE conference 2016, Milwaukee, WI, USA, 2016.

[18] C. Spiewak, Md R. Islam, M. H. Rahman, "Design with Integrated EMG Control of a 5DoF Robotic Assistive Device for Hand Rehabilitation (RAD-HR)," UWM's CEAS 2017 poster competition, Milwaukee, WI, USA, 2017.

[19] B. Brahim, C. Ochoa Luna, M. Saad, Md A.-Uz-Zaman, Md R. Islam, M. H. Rahman, "A new Adaptive SuperTwisting Control for an Exoskeleton Robot with Dynamic Uncertainties," Great Lakes Biomedical Conference 2017, Milwaukee, WI, USA, 2017.

[20] Md Assad-Uz-Zaman, Md Rasedul Islam, Brahim Brahmi, Miguel Garcia, Mohammad Rahman, M Saad, (2017), "Motion Control Of A Robotic Prosthetics With Skin Surface Emg Signals" UWM's CEAS 2017 poster competition, Milwaukee, WI,USA.

[21] C. Spiewak, Md R. Islam, M. Habibur Rahman, M. Saad, "Myo Signal Based Control of a Robotic Assistive Device for Hand Rehabilitation," Great Lakes Biomedical Conference 2017, Milwaukee, WI, USA.

[22] J. J. Craig, Introduction to robotics: mechanics and control. Upper Saddle River, N.J., Pearson/Prentice Hall, 2005.

[23] SoftBank Robotics, (November 12, 2017). [Online]. Available: http://doc.aldebaran.com/index.html

[24] D. A. Winter, Biomechanics and motor control of human movement, 2nd ed. New York: J. Wiley, xvi, p. $277,1990$.

[25] E. Pot, J. Monceaux, R. Gelin and B. Maisonnier, "Choregraphe: a graphical tool for humanoid robot programming," RO-MAN 2009 - The 18th IEEE International Symposium on Robot and Human Interactive Communication, Toyama, 2009, pp. 46-51. DOI: 10.1109/ROMAN.2009.5326209. 